\title{
Lumbosacral Transitional Vertebra Diagnosed on 99mTc-Methylene Diphosphonate SPECT/CT
}

\author{
Mohammad A. Kassir, Zaid Al-faham, Nicholas Abel, and Helena R. Balon \\ Department of Diagnostic Radiology and Molecular Imaging, Oakland University William Beaumont School of Medicine and \\ Beaumont Health System, Royal Oak, Michigan
}

Lumbosacral transitional vertebra (LSTV) is a congenital anomaly of the lumbosacral junction. The association between back pain and LSTV is controversial; however, in our patient the symptoms localized to a hemisacralized left transverse process of L5. LSTV should be included in the differential diagnosis in young patients with lower back pain, and scintigraphic imaging should be considered for diagnostic purposes.

Key Words: bone; SPECT; SPECT/CT; Bertolloti syndrome; lumbosacral transitional vertebra

J Nucl Med Technol 2015; 43:137-138

DOI: 10.2967/jnmt.114.146639

$\mathbf{L}$

umbosacral transitional vertebra (LSTV) is a congenital malformation of the lumbosacral spine that can present as lumbarization of the first sacral vertebra or sacralization of the lowest lumbar vertebra. Association of LSTV with lower back pain is known as Bertolotti syndrome and is thought to be related to increased stress at the lumbosacral articulation. LSTV is usually diagnosed on lumbar radiography, CT, or MR imaging. ${ }^{99 \mathrm{~m}} \mathrm{Tc}-\mathrm{methylene}$ diphosphonate SPECT bone scanning can be of additional value, as it helps determine whether there is stress at the articulation between the transitional vertebra and the sacrum.

\section{CASE REPORT}

A 16-y-old female presented with lower back pain for 8 mo. Her only reported medical history was left renal agenesis. She was a cheerleader but denied history of recent trauma. A bone scan was requested for suspected pars interarticularis defect. Whole-body bone scintigraphy was performed approximately $3 \mathrm{~h}$ after intravenous injection of $762 \mathrm{MBq}(20.6 \mathrm{mCi})$ of ${ }^{99 \mathrm{~m}} \mathrm{Tc}-\mathrm{methylene}$ diphosphonate. Planar images showed a focus of increased radiotracer activity in the left lumbosacral area overlying the left sacroiliac joint (Fig. 1). Because this pattern was considered atypical

\footnotetext{
Received Aug. 2, 2014; revision accepted Oct. 23, 2014.

For correspondence contact: Mohammad A. Kassir, Beaumont Health System, 3601 W. 13 Mile Rd., Royal Oak, MI 48703.

E-mail: mohammad.kassir@beaumont.edu

Published online Feb. 5, 2015.

COPYRIGHT (c) 2015 by the Society of Nuclear Medicine and Molecular Imaging, Inc.
}

for pars interarticularis fracture, we obtained another planar image with a marker placed over the area of pain and additional SPECT with low-dose CT imaging of the lumbosacral spine, instead of our routine protocol for pars interarticularis defect (SPECT without CT). The SPECT/CT images localized the focus of increased radiotracer activity to a hemisacralized left transverse process of L5 (Fig. 2). The CT appearance was consistent with a type IIa LSTV.

\section{DISCUSSION}

The lumbosacral junction often exhibits a transitional vertebra with sacralization of the lower-most lumbar segment or lumbarization of superior-most sacral segment. The Castellvi system is most commonly used for morphologic classification of LSTV (I). It defines types I-IV, with type I characterized by an enlarged $(>19 \mathrm{~mm})$ transverse process, type II representing vertebra with an incompletely fused broad transverse process forming a diarthrodial joint with the sacrum, and type III having complete fusion of the transverse process with the sacrum. Under each type, unilateral findings are denoted as subtype a, and bilateral findings are denoted $b$. Type IV consists of a unilateral complete fusion with contralateral incomplete fusion (type III on one side and type II on the other).

LSTV can be diagnosed using several imaging modalities. Historically, an anteroposterior radiograph of the lumbosacral junction angled $30^{\circ}$ cranially, known as a Ferguson radiograph, was the best study for evaluating transitional vertebrae (2). However, with the widespread use of CT and MR imaging, LSTV is now usually incidentally discovered on CT or MR imaging done for workup of back pain or for other reasons. Low-back pain associated with a LSTV may arise

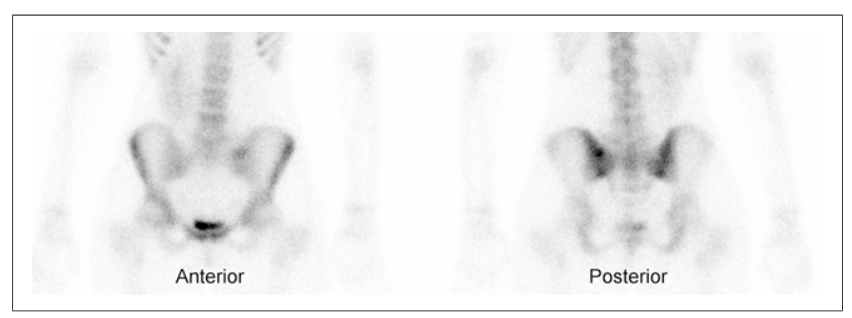

FIGURE 1. Anterior and posterior spot images of postvoid pelvis show focus of increased radiotracer activity in left lumbosacral area overlying left sacroiliac joint. Marker used to denote site of pain correlated closely to focus of increased radiotracer intensity (not shown). 
FIGURE 2. SPECT (A), low-dose CT (B), and hybrid SPECT/CT (C) images of lower lumbar spine and pelvis reveal hemisacralized left transverse process at level L5. Hybrid SPECT/CT image shows that focus of increased radiotracer activity corresponds to hemisacralized left transverse process of L5.

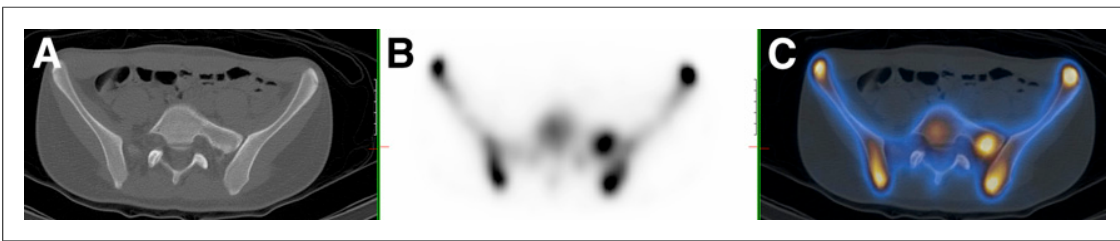

from the level above the transition, the contralateral facet when unilateral, or the anomalous articulation when present (3). In addition to standard radiologic modalities, nuclear medicine studies can be useful in the workup of LSTV.

SPECT can play an important role in the evaluation of patients with LSTV and lower back pain. Although the presence of a transitional vertebra can be established with plain film radiography, CT, or MR imaging, demonstration of stress at the transverse-sacral articulation is best accomplished using bone scanning with SPECT (4). The finding of intense radiotracer uptake at the transverse-sacral articulation in patients with LSTV seems to correlate with lower back pain symptoms. This is because the increase in radiotracer uptake will identify sites of increased osseous metabolic activity at the LSTV articulation site (5). These findings suggest that SPECT imaging can be a good tool for determining whether lower back pain in patients with LSTV is caused by the transitional vertebra or attributable to other causes. We believe hybrid SPECT/CT imaging improves the diagnostic capabilities of the bone scan by localizing the increased osseous activity more accurately.

\section{CONCLUSION}

LSTV is a congenital malformation of the lumbosacral spine. It is mostly asymptomatic; however, it can present with lower back pain. Bertolotti syndrome should always be included in the differential diagnosis of lower back pain in young patients. LSTV can be diagnosed using several imaging modalities including radiography, MR imaging, and CT. This case shows that a whole-body bone scan with hybrid SPECT/CT imaging of the area of pain can be a tool to help diagnose LSTV.

\section{DISCLOSURE}

No potential conflict of interest relevant to this article was reported.

\section{REFERENCES}

1. Castellvi AE, Goldstein LA, Chan DP. Lumbosacral transitional vertebrae and their relationship with lumbar extradural defects. Spine (Phila Pa 1976). 1984;9:493-495.

2. Hughes RJ, Saifuddin A. Imaging of lumbosacral transitional vertebrae. Clin Radiol. 2004;59:984-991.

3. Konin GP, Walz DM. Lumbosacral transitional vertebrae: classification, imaging findings, and clinical relevance. AJNR. 2010;31:1778-1786.

4. Connolly LP, d'Hemecourt PA, Connolly SA, Drubach LA, Micheli LJ, Treves ST. Skeletal scintigraphy of young patients with low-back pain and a lumbosacral transitional vertebra. J Nucl Med. 2003;44:909-914.

5. Pekindil G, Sarikaya A, Pekindil Y, Gültekin A, Kokino S. Lumbosacral transitional vertebra articulation: evaluation by planar and SPECT bone scintigraphy. Nucl Med Commun. 2004;25:29-37. 